\title{
Energy elastic effects and the concept of temperature in flowing polymeric liquids
}

\section{Journal Article}

\section{Author(s):}

Hütter, Markus; Luap, Clarisse; Öttinger, Hans Christian (I)

Publication date:

2009-04

Permanent link:

https://doi.org/10.3929/ethz-b-000021242

Rights / license:

In Copyright - Non-Commercial Use Permitted

Originally published in:

Rheologica Acta 48(3), https://doi.org/10.1007/s00397-008-0318-8 


\title{
Energy elastic effects and the concept of temperature in flowing polymeric liquids
}

\author{
Markus Hütter • Clarisse Luap • \\ Hans Christian Öttinger
}

Received: 1 April 2008 / Accepted: 16 September 2008 / Published online: 30 October 2008

(C) Springer-Verlag 2008

\begin{abstract}
The incorporation of energy elastic effects in the modeling of flowing polymeric liquids is discussed. Since conformational energetic effects are determined by structural features much smaller than the end-toend vector of the polymer chains, commonly employed single conformation tensor models are insufficient to describe energy elastic effects. The need for a local structural variable is substantiated by studying a microscopic toy model with energetic effects in the setting of a generalized canonical ensemble. In order to examine the dynamics of flowing polymeric liquids with energy elastic effects, a thermodynamically admissible set of evolution equations is presented that accounts for the evolution of the microstructure in terms of a slow tensor, as well as a fast, local scalar variable. It is demonstrated that the temperature used in the definition of the heat flux is directly related to the Lagrange multiplier of the microscopic energy in the generalized canonical partition function. The temperature equation is discussed with respect to, first, the dependence of the heat capacity on the polymer conformation and, second, the possibility to measure experimentally the effects of the conformational energy.
\end{abstract}

Keywords Energy elastic effect • Local structural variable $\cdot$ Viscoelastic fluid . Nonequilibrium thermodynamics • Generalized canonical ensemble

M. Hütter $(\bowtie) \cdot$ C. Luap · H. C. Öttinger

Department of Materials, Polymer Physics,

ETH Zürich, Wolfgang-Pauli-Str. 10,

8093 Zürich, Switzerland

e-mail: markus.huetter@mat.ethz.ch

\section{Introduction}

Entropy elasticity of rubbers (Treloar 1975) serves as a starting point for most of the current models to describe the flow of polymeric liquids, while consideration of energetic effects is scarce. Such exclusive subscription to entropy elasticity derives from the following contrast. The slowest degrees of freedom, which are of primary importance for the rheology under slow and moderate deformation rates, describe large-scale features in the polymeric liquid, whereas the energetic effects depend on local aspects of the chain conformations that have much faster relaxation processes. Furthermore, in particular, the variables describing the large scales lead to significant contributions to the entropy due to the large number of corresponding microstates. Nevertheless, it has recently been demonstrated using configurationally biased Monte Carlo simulations that energetic effects of the polymer conformation indeed become relevant, particularly at large deformations, which supports the significance of fast local degrees of freedom (Mavrantzas and Theodorou 1998; Ionescu et al. 2008b). For these reasons, it is interesting to discuss the possible ramifications of energy elasticity on the flow behavior of polymeric liquids. In order to arrive at a model suitable for describing conformational energetic effects, the following questions must be addressed: (1) What is a suitable choice of variables to capture energy elastic effects? (2) How can energetic and entropic contributions be distinguished properly in the thermodynamic potentials?

The proper choice of variables is of fundamental importance in modeling complex fluids, as it is often stated and also well known. The key aspect of the word "proper" means that the set of variables must be 
sufficiently detailed to capture the physics one is interested in describing. Since polymer chains depart significantly from equilibrium upon the application of flow, structural variables are chosen to capture that deviation (Bird et al. 1977; Larson 1988), including the distribution function $p(\boldsymbol{R})$ of the end-to-end vector $\boldsymbol{R}$ between chain ends or the second moment $\boldsymbol{c}=\langle\boldsymbol{R} \boldsymbol{R}\rangle$, the distribution function $p(\boldsymbol{n}, s)$ of the tangent vector $\boldsymbol{n}$ and label $s$ along the primitive path in the reptation model, or the local chain stretch $\lambda$ (Öttinger 1999). Although these choices differ in their applicability to polymeric solutions and melts, respectively, a feature common to them is that they are usually employed to describe entropy elasticity only, while energetic effects are neglected, a preference that may be traced back to the works of Astarita (1974), Astarita and Sarti (1976), and Sarti and Esposito (1977). Exceptions in this regard are some applications of conformation tensor models, in which the entropic spring for $\boldsymbol{R}$ is replaced by a spring with mixed entropic and energetic character (Braun and Friedrich 1990; Peters and Baaijens 1997; Wapperom and Hulsen 1998; Dressler et al. 1999). Such an extension is certainly admissible if $\boldsymbol{c}$ is to remain the only relevant structural variable in the model. However, by virtue of the physical origin of energetic effects, e.g., as described by the rotational isomeric state (RIS) model (Flory 1988; Mattice and Suter 1994) or the local packing of chain segments, it seems that structural features on scales much finer than the end-to-end vector must be resolved to represent the energetics appropriately. Ignoring the existence of an independent local variable and instead linking it statically to the conformation tensor seems inadequate and would be a severe restriction of the physics, in particular with regards to the dynamics, since the more local the structural feature is, the more rapid is its associated relaxation processes (Bird et al. 1977; Larson 1988). Local structural variables have been introduced previously for the modeling of flowing polymeric liquids, e.g., in some versions of the reptation model (Ianniruberto and Marrucci 2001; Marrucci and Ianniruberto 2003) and in the pompon model for melts of multiply branched polymers (McLeish and Larson 1998; Öttinger 2001; Soulages et al. 2006). While, in both of these examples, the scalar structural variable represents local chain (segment) stretch that relaxes substantially faster than the tensorial variable, the step to incorporate energy elasticity has not been taken in full generality by Öttinger $(2002,2005)$.

The distinction of energetic and entropic effects based on a thermodynamic potential in nonequilibrium situations is more involved than it may seem at first sight. To address the energy-entropy separation in equilibrium thermodynamics, one usually departs from the free energy $F(T, X)$ with absolute temperature $T$ and extensive variables $X$, based on which, the quantity $E=F-T \partial F /\left.\partial T\right|_{X}$ is identified with the energy of the system. This identification can be substantiated with statistical mechanics since, in the canonical ensemble, $E$ is the ensemble average of the microscopic Hamiltonian (Reichl 1980; Landau and Lifshitz 1980; Callen 1985). Clearly, this procedure for identification of energetic and entropic contributions rests on the clear concept of temperature, which is, however, a cumbersome one in beyond equilibrium situations (Grmela 1998; Casas-Vázquez and Jou 2003). Therefore, one must examine critically the ways to achieve the energy-entropy separation based on outof-equilibrium Helmholtz free energy expressions, as implicitly invoked, e.g., in Braun and Friedrich (1990), Peters and Baaijens (1997), Wapperom and Hulsen (1998), and Dressler et al. (1999). In order to clarify the meaning of $T$ as a variable out of equilibrium similar to the scenario in equilibrium, one can envision that, also out-of-equilibrium, a statistical mechanics treatment must come to the aid to bring clarification. To our knowledge, such steps have not yet been taken in literature.

In order to address these issues, the manuscript is organized as follows: We start with a microscopically motivated toy model that includes conformational energetic effects in terms of different RIS states (Flory 1988; Mattice and Suter 1994). For this model, both the coarse-grained conformational energy and the conformational entropy are calculated in a generalized canonical ensemble and expressed in terms of the conformation tensor $\boldsymbol{c}$ and a local structural variable $\lambda$. In order to study the dynamic behavior of such a system under flow, the general equation for the nonequilibrium reversible-irreversible coupling (GENERIC) framework (Grmela and Öttinger 1997; Öttinger and Grmela 1997; Öttinger 2005) is then used to formulate a closed set of evolution equations that includes the dynamics of $c$ and $\lambda$, apart from the usual hydrodynamic variables. The resulting model is discussed with regards to the ramifications of conformational energetic effects and the use of a temperature-like dynamic variable, and is illustrated for the previously introduced toy model.

\section{Toy model}

With the aid of a simple toy model, which can be treated analytically, it will be shown that energetic effects of the polymer conformation are described with a structural variable that resolves length scales significantly more 
local than what is characterized by the conformation tensor. To that end, a generalized canonical ensemble is considered to relate the microscopic understanding about the chain with the level of a thermodynamic state function, valid also out of equilibrium. For the sake of keeping the model tractable, we consider only intrachain energetic effects. Possible extensions are addressed in the "Summary and perspectives" section.

While in the usual canonical ensemble, the average value of the energy $H$ is conveniently controlled by the Lagrange multiplier $\beta=1 /\left(k_{\mathrm{B}} T\right)$, out-of-equilibrium situations of polymeric systems involve further (extensive) variables $\left\{X_{i}\right\}_{i=1, \ldots, k}$, the averages of which can be controlled by corresponding Lagrange multipliers $\left\{\alpha_{i}\right\}_{i=1, \ldots, k}$. As an example, one structural variable can be the dyadic of the end-to-end vector, $X_{1}=\boldsymbol{R} \boldsymbol{R}$. The generalized canonical partition function can formally be written as (Öttinger 2005)

$Z\left(\beta,\left\{\alpha_{i}\right\}\right)=\sum_{\mathrm{PS}} e^{-\beta H-\sum_{i=1}^{k} \alpha_{i} X_{i}}$,

where the summation (integration) runs over all of the available phase space (PS). Based on that partition function, one can derive expressions for the entropy $S$ and for the averages of $H$ and $X_{i}$,

$S=k_{\mathrm{B}}\left(\ln Z+\beta\langle H\rangle+\sum_{i=1}^{k} \alpha_{i}\left\langle X_{i}\right\rangle\right)$,

$E \equiv\langle H\rangle=-\left.\frac{\partial \ln Z}{\partial \beta}\right|_{\alpha_{i}}$,

$\left\langle X_{i}\right\rangle=-\left.\frac{\partial \ln Z}{\partial \alpha_{i}}\right|_{\beta, \alpha_{k}, k \neq i}$,

all expressed as functions of $\beta$ and $\left\{\alpha_{i}\right\}_{i=1, \ldots, k}$. With the access to the Lagrange parameters $\alpha_{i}$ in the partition function Eq. 1, one has a handle on the averages $\left\langle X_{i}\right\rangle$, which means that out-of-equilibrium situations can be generated in this way, as used in Monte Carlo simulations of polymer melts (Mavrantzas and Theodorou 1998; Mavrantzas and Öttinger 2002; Baig and Mavrantzas 2007; Ionescu et al. 2008b). Using a generalized canonical partition function of the form Eq. 1, it is straightforward to show that the following, model-independent, relations between the entropy and energy defined in Eq. 2 hold,

$$
\begin{aligned}
& \left.\frac{\partial\left(S / k_{\mathrm{B}}\right)}{\partial \beta}\right|_{\left\langle X_{i}\right\rangle}-\left.\beta \frac{\partial E}{\partial \beta}\right|_{\left\langle X_{i}\right\rangle}=0, \\
& \left.\frac{\partial}{\partial\left\langle X_{i}\right\rangle}\left(\frac{S}{k_{\mathrm{B}}}-\beta E\right)\right|_{\beta,\left\langle X_{k}\right\rangle, k \neq i}=\alpha_{i},
\end{aligned}
$$

where it must be noted that the averages $\langle X\rangle$ rather than the Lagrange multipliers $\alpha$ are held constant upon differentiation.

As a specific example for illustrating conformational energetic effects, we consider a single linear polymer chain composed of $N$ (united) atoms with variable bond lengths, bond angles, and torsion angles. To render analytic calculations for this toy model feasible and transparent, we assume that the conformation of such a chain can be described by (1) well-defined RIS (Flory 1988; Mattice and Suter 1994) with fixed bond length, fixed bond angle, and discrete values for the torsion angle, and (2) local vibrations around each RIS state. While the potentials describing the bond length and bond angle degrees of freedom shall have a single minimum each, the torsional degree of freedom is assumed to take three states (local minima): a trans state $(t)$ of zero energy by definition, and two gauche states $\left(g^{+}\right.$ and $g^{-}$) with a energy penalty of $\epsilon$, each with respect to the trans state. This model is representative of polyethylene, and relates to the potentials for bond length, bond angle, and bond torsion as used in realistic united atom simulations (Paul et al. 1995; In 't Veld et al. 2006). The number of gauche states along the chain are denoted by $n$, and hence, $\epsilon n$ is the instantaneous conformational energy of a chain.

The energy and entropy for the toy model defined above can be calculated as follows. In order to describe a single polymer chain in situations out of equilibrium, we introduce $\alpha$ and $\mu$ as the Lagrange multipliers to the structural variables $X_{1}=\boldsymbol{R} \boldsymbol{R}$ and $X_{2}=n$, respectively, and calculate the generalized canonical partition function $Z(\beta, \boldsymbol{\alpha}, \mu)$. It can be shown (see Appendix A for details) that the expressions for the energy and entropy of a single chain (sc) of length $N$ in terms of $\beta$, the conformation tensor $\boldsymbol{c}=\langle\boldsymbol{R} \boldsymbol{R}\rangle$, and the (average) number of gauche states $n$ assume the form

$$
\begin{aligned}
E^{\mathrm{sc}}(\beta, \boldsymbol{c}, n)= & \left(F_{\mathrm{loc}}(\beta, n)+\beta \frac{\partial F_{\mathrm{loc}}(\beta, n)}{\partial \beta}\right)+\epsilon n \\
S^{\mathrm{sc}}(\beta, \boldsymbol{c}, n)= & k_{\mathrm{B}} \beta^{2} \frac{\partial F_{\mathrm{loc}}(\beta, n)}{\partial \beta}+S_{\mathrm{conf}}(n) \\
& +\frac{k_{\mathrm{B}}}{2}\left[3-\operatorname{tr}\left(\boldsymbol{c} / c_{0}(n)\right)+\ln \operatorname{det}\left(\boldsymbol{c} / c_{0}(n)\right)\right],
\end{aligned}
$$

where $F_{\text {loc }}$ represents the local Helmholtz free energy (Eq. 34) accounting for the local vibrations around the RIS conformations with $n$ gauche states. As discussed in Appendix A, the $n$-dependence of $F_{\text {loc }}$ can be neglected if the form of the atomistic potentials around a RIS conformation with $n$ gauche states is effectively independent of $n$, which, e.g., is reasonably 
well satisfied by the torsion potential for polyethylene. The conformational entropy is given by

$S_{\text {conf }}(n)=k_{\mathrm{B}} \ln \left(2^{n}\left(\begin{array}{c}N-2 \\ n\end{array}\right)\right)$,

which counts the number of possible RIS conformations with $n$ of the $N-2$ torsional degrees of freedom being in the (twofold degenerate) gauche state. In the entropy Eq. $4 \mathrm{~b}$, the quantity $c_{0}$ is related to the distribution of the end-to-end vector $\boldsymbol{Q}$ of all RIS conformations for given $n,\langle\boldsymbol{Q} \boldsymbol{Q}\rangle=c_{0}(n) \mathbf{1}$. Keeping constant the bond length $l$ and the bond angle $\vartheta$, and with torsion angle $\varphi$, one can write for long chains of length $N$ (Flory 1988)

$$
\begin{aligned}
c_{0}(n) & =\frac{(N-1) l^{2}}{3} C_{\infty} \\
& =\frac{(N-1) l^{2}}{3}\left(\frac{1+\cos \vartheta}{1-\cos \vartheta}\right)\left(\frac{1+\langle\cos \varphi\rangle}{1-\langle\cos \varphi\rangle}\right),
\end{aligned}
$$

where it has been assumed that the torsion angles are independent from each other and that the energy of a single torsion angle $\varphi$ is symmetric in $\varphi$ around $\varphi=0$. As the torsion angle in the RIS model can assume only three distinct values, e.g., for polyethylene $\varphi_{\text {trans }}=0$ and $\varphi_{\text {gauche }} \simeq \pm 2$, one has

$$
\langle\cos \varphi\rangle=\frac{n}{N-2} \cos \varphi_{\text {gauche }}+\left(1-\frac{n}{N-2}\right) \cos \varphi_{\text {trans }},
$$

i.e., the dependence of $c_{0}$ on $n$ and $N$ for long chains is of the form $f(n / N) N$.

The total energy $E^{\text {sc }}$ splits into a local and a conformational contribution depending on $n$, while the entropy $S^{\text {sc }}$ consists of a local term and two conformational ones, depending on $n$ and $\boldsymbol{c}$, respectively. Given the weak dependence of $F_{\text {loc }}$ on $n$, the influence of $n$ on the local energy and local entropy, respectively, becomes negligible. Identifying the different contributions is useful for interpreting the deformation of the polymeric system. For example, the exchange between local and conformational entropy explains the heating of an adiabatically stretched rubber. Similar insights can be expected from accounting for both forms of energy, local and conformational. The absence of a $\boldsymbol{c}$ dependent contribution to the energy is noteworthy and highlights the effect of using the local structural variable $n$.

Since the parameters $\boldsymbol{\alpha}$ and $\mu$ in the generalized canonical ensemble have been introduced to drive the system out of equilibrium, it is now interesting to express them for the specific toy model in terms of the variables $(\beta, \boldsymbol{c}, n)$. Application of the identity Eq. $3 b$ to the single chain energy and entropy Eq. 4 leads to

$\boldsymbol{\alpha}=\frac{1}{2}\left(\boldsymbol{c}^{-1}-\frac{1}{c_{0}(n)} \mathbf{1}\right)$,

$\mu=-\beta \epsilon+\frac{\partial\left(S_{\mathrm{conf}} / k_{\mathrm{B}}\right)}{\partial n}-\beta \frac{\partial F_{\mathrm{loc}}}{\partial n}-\boldsymbol{c}: \alpha \frac{d \ln c_{0}}{d n}$.

In equilibrium, $\boldsymbol{\alpha}=\mathbf{0}$, one thus finds $\boldsymbol{c}_{\mathrm{eq}}=c_{0}(n) \mathbf{1}$, which highlights the meaning of the function $c_{0}(n)$. Equilibrium furthermore requires $\mu=0$, from which one obtains, by virtue of Eq. $7 \mathrm{~b}$, a relation of the form $n_{\mathrm{eq}}(\beta)$. This in turn leads to the $\beta$-dependent conformation tensor $\boldsymbol{c}_{\mathrm{eq}}=c_{0}\left(n_{\mathrm{eq}}(\beta)\right) \mathbf{1}$ of the polymer chain at equilibrium (see "Implications of the model" section below for details). On the other hand, if only the local variable $n$ is equilibrated $(\mu=0)$ but the large-scale variable $\boldsymbol{c}$ is not $(\boldsymbol{\alpha} \neq \mathbf{0})$, one finds from Eq. $7 \mathrm{~b}$ by neglecting $\partial F_{\text {loc }} / \partial n$ the implicit expression for $n / N$ in the long chain limit, $N-2 \simeq N$,

$\frac{n}{N}=\frac{2 \mathrm{e}^{-\beta \epsilon-Y}}{1+2 \mathrm{e}^{-\beta \epsilon-Y}}$,

$Y \equiv \frac{1}{2}\left[3-\operatorname{tr}\left(\boldsymbol{c} / c_{0}(n)\right)\right] \frac{d \ln c_{0}(n)}{d n}$.

In view of the form Eq. 6 for $c_{0}$, one observes that $d \ln c_{0}(n) / d n$ scales in the long chain limit with fixed $n / N$ as $N^{-1}$. Therefore, it is reasonable to assume $Y \ll 1$, which in turn leads to the probability of gauche states

$\frac{n}{N} \simeq \frac{2 \mathrm{e}^{-\beta \epsilon}}{1+2 \mathrm{e}^{-\beta \epsilon}}\left(1-\frac{Y}{1+2 \mathrm{e}^{-\beta \epsilon}}\right)$,

i.e., linear in the out-of-equilibrium term $3-\operatorname{tr}\left(\boldsymbol{c} / c_{0}\right)$. In order to arrive at an explicit equation for $n / N$ correct to first order in $3-\operatorname{tr}\left(\boldsymbol{c} / c_{0}\right)$, one can replace in $Y$ the occurrence of $n$ by its value for $\boldsymbol{c}=c_{0} \mathbf{1}$.

After these discussions, we proceed with comparing our expressions (Eq. 4) with the ones used in the literature. The $\boldsymbol{c}$-dependent terms in the entropy are the well known result found in literature (Sarti and Marrucci 1973; Dressler et al. 1999). However, in Sarti and Marrucci (1973), the unperturbed size of the chain depends on the temperature, i.e. $c_{0}(T)$, while we have explicit access to the number of gauche states and, hence, $c_{0}(n)$. As discussed above, only in the limit of an equilibrated number of gauche states $n$ (i.e., for $\mu=0)$ do we observe the effect of $\beta$ on $c_{0}$. The contributions $\epsilon n$ and $S_{\text {conf }}$ in the energy Eq. 4a and entropy Eq. 4b, respectively, are new conformational contributions. In contrast, in Dressler et al. (1999), a term proportional to $\operatorname{trc}$ is added to the energy to represent energetic effects. Specifically, the following 
conformation-dependent contributions to the energy and entropy are used, here expressed for a single chain,

$$
\begin{aligned}
& \left.E^{\text {sc,Dressler }}(T, \boldsymbol{c})\right|_{\text {conf }}=\frac{1}{2}\left(K(T)-T \frac{d K(T)}{d T}\right) \operatorname{tr}(\boldsymbol{c}),(9 \mathrm{a}) \\
& \left.S^{\text {sc,Dressler }}(T, \boldsymbol{c})\right|_{\text {conf }}=\frac{1}{2}\left[-\frac{d K(T)}{d T} \operatorname{tr}(\boldsymbol{c})+k_{\mathrm{B}} \ln \operatorname{det}(\boldsymbol{c})\right],
\end{aligned}
$$

with the overall chain spring constant $K(T)$ with temperature $T$. In their case, energetic effects arise as the dependence of $K$ on $T$ is nonlinear. However, in the light of the microscopic origin of energetic effects (e.g., in our toy model), it seems to us more desirable to capture these local effects with a local structural variable, as exemplified with our toy model. With regard to the approximate form of gauche states (Eq. 8c), it is clear that, in our approach, the conformational energy $\epsilon n$ can be expressed as a term linear in trc only under special circumstances, namely, if $n$ is equilibrated and if the effect of $\operatorname{tr} c$ on $n$ is weak. In particular, with the approximation mentioned after Eq. 8c, the energy term linear in $\operatorname{tr} c$ assumes the form

$E^{\mathrm{sc}}=-\frac{1}{2} \frac{d \ln c_{0}}{d \beta} \operatorname{tr}\left(\boldsymbol{c} / c_{0}\right)+\ldots$,

where the dots stand for terms independent of $\boldsymbol{c}$. The derivative of $c_{0}$ must be understood as a derivative with respect to $n$ at $Y=0$, multiplied by the derivative of $n$ from Eq. 8c at $Y=0$ with respect to $\beta$. It is comforting to note that the form Eq. 10 is identical to the corresponding contribution contained in Eq. 9a when using their relation $K(T)=k_{\mathrm{B}} T / c_{0}(T)$ (Dressler et al. 1999; Ionescu et al. 2008a), under the condition $T=$ $\left(k_{\mathrm{B}} \beta\right)^{-1}$. It will be shown below in the "Discussion" that this condition is indeed satisfied; see Eq. 22.

\section{Formulation of the dynamics}

\section{Strategy}

Motivated by the above toy model, the use of a local structural variable in addition to the conformation tensor is promising for addressing the effect of conformational energy on the behavior of polymers. In order to examine the flow behavior, we aim at formulating a thermodynamically admissible, closed set of evolution equations including a scalar and tensorial variable, $\lambda$ and $\boldsymbol{c}$, for describing the conformation of the polymer chains. To keep this development from being restricted to the toy model, we do not imply that $\lambda=n$, and we also use the most general form for the energy and entropy as functions of the structural variables. Afterwards, the ramifications of the toy model can be studied as a special case of the general model.

As guard-rail to formulate the dynamic model, we choose the GENERIC framework by Grmela and Öttinger (Grmela and Öttinger 1997; Öttinger and Grmela 1997; Öttinger 2005) of beyond-equilibrium thermodynamics. In regard to the topic of this paper, this method is particularly suitable for two reasons. First, it is applicable to nonisothermal situations, and second, the specification of generalized thermodynamic potentials, namely energy and entropy, is a key ingredient that allows for a large flexibility of the phenomena under consideration. In the following, we summarize only briefly those aspects of GENERIC that are of relevance for this paper.

When trying to formulate a model in that framework, the first step is to choose a set of variables, $\boldsymbol{x}$, that describes the situation of interest to sufficient detail. The time evolution of the variables $\boldsymbol{x}$ of an isolated system are then written in the form

$\frac{\partial \boldsymbol{x}}{\partial t}=\boldsymbol{L}(\boldsymbol{x}) \cdot \frac{\delta E}{\delta \boldsymbol{x}}+\boldsymbol{M}(\boldsymbol{x}) \cdot \frac{\delta S}{\delta \boldsymbol{x}}$,

where the two generators $E$ and $S$ are the total energy and entropy functionals in terms of the state variables $\boldsymbol{x}$, and $\boldsymbol{L}$ and $\boldsymbol{M}$ are certain matrices (operators). The two contributions to the time evolution of $\boldsymbol{x}$ generated by the total energy $E$ and the entropy $S$ are called the reversible and irreversible contributions, respectively. The operator $\boldsymbol{L}$ describes the kinematics, while the operator $\boldsymbol{M}$ accounts for a wealth of irreversible processes and, hence, contains material parameters such as diffusion coefficients, viscosities, relaxation times, and reaction constants. The matrix multiplications (symbol ·) imply not only summations over discrete indices but may also include integration over continuous variables, in particular for non-local field theories, and $\delta / \delta \boldsymbol{x}$ typically implies functional rather than partial derivatives.

The GENERIC structure also imposes certain conditions on the building blocks in Eq. 11. First, Eq. 11 is supplemented by the mutual degeneracy requirements

$$
\begin{aligned}
& \boldsymbol{L}(\boldsymbol{x}) \cdot \frac{\delta S}{\delta \boldsymbol{x}}=\mathbf{0}, \\
& \boldsymbol{M}(\boldsymbol{x}) \cdot \frac{\delta E}{\delta \boldsymbol{x}}=\mathbf{0},
\end{aligned}
$$

representing entropy and energy conservation upon reversible and irreversible dynamics, respectively. In addition, $\boldsymbol{L}$ must be antisymmetric, whereas $\boldsymbol{M}$ needs to be positive-semidefinite and Onsager-Casimir symmetric. As a result of all these conditions, one may show 
that the GENERIC Eq. 11 implies both the conservation of total energy, as well as a nonnegative entropy production. Finally, the GENERIC structure requires that the Poisson bracket associated to the operator $\boldsymbol{L}$ fulfills the Jacobi identity, which expresses the timestructure invariance of the reversible dynamics. The complementary degeneracy requirements, the symmetry properties, and the Jacobi identity are essential for formulating proper $\boldsymbol{L}$ - and $\boldsymbol{M}$-matrices when modeling concrete nonequilibrium problems. Various applications have shown that the two-generator idea and the criteria on the two matrices have strong implications (e.g., Öttinger and Grmela 1997; Öttinger 2002, 2005; Edwards and Öttinger 1997).

Choice of variables $\boldsymbol{x}$, generating functionals $E$ and $S$

The toy model in the previous section has illustrated that, in addition to a conformation tensor $\boldsymbol{c}$, a local structural variable, denoted by $\lambda$, is useful for capturing conformational energetic effects. Furthermore, in order to describe nonisothermal flow, we use the "hydrodynamic" variables momentum density $\boldsymbol{u}$, mass density $\rho$, and a yet unspecified thermal variable $\theta$. In view of the toy model and of capturing the fast vibrations that are unresolved by $\lambda$ and $\boldsymbol{c}$, the variable $\theta$ can be interpreted as related to the Lagrange parameter $\beta$, the local energy, or the local entropy. In summary, the full set of variables is thus given by

$\boldsymbol{x}=(\boldsymbol{u}, \rho, \theta, \boldsymbol{c}, \lambda)$.

The tensor $\boldsymbol{c}$ is interpreted as the average $\boldsymbol{c}=\langle\boldsymbol{R} \boldsymbol{R}\rangle$ introduced in the "Introduction." In other words, it is a tensor rather than a tensor density and shows upper convected Maxwell behavior, both of which will be of relevance when formulating the reversible dynamics. Note that, in the above choice of structural variables, it is implicit that there are no intermediate time scales between what is described by $c$ and $\lambda$, respectively. In other words, the following description is supposed to be reasonable for relatively short chains as far as qualitative aspects of the energetic effects are concerned. If one were interested in quantitative predictions, more variables would have to be included.

The functionals for the total energy and the total entropy in terms of the variables $\boldsymbol{x}$ are written in the form

$$
\begin{aligned}
& E=\int\left(\frac{\boldsymbol{u}^{2}}{2 \rho}+e_{\mathrm{t}}(\rho, \theta, \boldsymbol{c}, \lambda)\right) d^{3} r, \\
& S=\int s_{\mathrm{t}}(\rho, \theta, \boldsymbol{c}, \lambda) d^{3} r,
\end{aligned}
$$

with the only assumption that the total energy is the sum of a kinetic contribution plus an internal energy term, the latter being independent of the momentum density. In both expressions, the symbols $e_{\mathrm{t}}$ and $s_{\mathrm{t}}$ stand for both local and conformational effects. With the set of variables Eq. 13 and by keeping the functions $e_{\mathrm{t}}$ and $s_{\mathrm{t}}$ general, the evolution equations developed in Öttinger $(2002,2005)$ are extended as to achieve highest flexibility in modeling conformational energetic effects.

For the microscopic toy model introduced above, one can choose $\theta=\left(k_{\mathrm{B}} \beta\right)^{-1}$ and $\lambda=n($ or $\lambda=n / N)$. The functions $e_{\mathrm{t}}$ and $s_{\mathrm{t}}$ are then given by multiplication of the single chain expressions in Eq. 4 by the number density of polymer chains, $\rho / M_{\mathrm{w}}$, where $M_{\mathrm{w}}$ denotes the molecular weight of each polymer.

\section{Operators $\boldsymbol{L}$ and $\boldsymbol{M}$}

The operators $\boldsymbol{L}$ and $\boldsymbol{M}$, which are involved in the reversible and irreversible contributions to the evolution equations (Eq. 11), are discussed in detail in Appendix B. The main points for their specification are the following.

For $\boldsymbol{L}$, it is observed, first, that the reversible contributions to the full evolution equations of the variables $\boldsymbol{x}$ are of purely kinematic origin, and hence, directly related to the velocity field $\boldsymbol{v}$. Second, the reversible evolution of the conformation tensor $\boldsymbol{c}$ and of the scalar $\lambda$ is assumed to be given by

$$
\begin{aligned}
& \left.\partial_{t} \boldsymbol{c}\right|_{\mathrm{rev}}=-\boldsymbol{v} \cdot \nabla \boldsymbol{c}+\boldsymbol{\kappa} \cdot \boldsymbol{c}+\boldsymbol{c} \cdot \boldsymbol{\kappa}^{T}, \\
& \left.\partial_{t} \lambda\right|_{\mathrm{rev}}=-\boldsymbol{v} \cdot \nabla \lambda+\boldsymbol{\kappa}: \boldsymbol{g},
\end{aligned}
$$

with the transpose velocity gradient $\kappa=(\nabla v)^{T}$. In other words, $\boldsymbol{c}$ shows upper convected Maxwell behavior, and the evolution of the scalar $\lambda$ is coupled to $\boldsymbol{c}$, where $\boldsymbol{g}$ is a function of $\lambda$ and $\boldsymbol{c}$. Since $\lambda$ is a scalar, such coupling is required to describe, for example, orientation-dependent local chain stretching upon the application of flow. It can indeed be shown that a Poisson operator $\boldsymbol{L}$ can be found that satisfies all the GENERIC conditions, provided that the function $\boldsymbol{g}(\boldsymbol{c}, \lambda)$ is of the specific form Eqs. 44-47.

In order to construct $\boldsymbol{M}$, we include as irreversible effects anisotropic heat conduction, and the relaxation of the conformational variables $\boldsymbol{c}$ and $\lambda$. For simplicity, anisotropic viscous flow and slip effects on the conformation tensor, as occurring in Schowalter derivatives, are not included. Due to the restriction to heat conduction and structural relaxation, only three thermodynamic driving forces are relevant. Correspondingly, there are three thermodynamic fluxes, namely, the heat 
flux, the rate of relaxation of $\boldsymbol{c}$, and the rate of relaxation of $\lambda$. Transport coefficients are then introduced to relate the forces to the fluxes. In particular, the following transport coefficients come into play: a second rank tensor $\boldsymbol{k}$ characterizes anisotropic thermal conductivity (Schieber et al. 2004), and a fourth rank tensor $\boldsymbol{\Lambda}$ and a scalar rate $R$ describe the relaxation of the conformation tensor $c$ and scalar variable $\lambda$, respectively. Crosseffects in the force-flux relations are also possible. For example, the relaxation of $\boldsymbol{c}$ can give rise to a heat flux, as will be quantified below by the transport coefficient $Z_{1}$ of tensorial rank three. Furthermore, the relaxation of $\lambda$ could affect the relaxation of $\boldsymbol{c}$, this coupling being described below by the transport coefficient $\boldsymbol{Z}_{2}$ of tensorial rank two. Any ansatz for the quantities $\boldsymbol{k}, \boldsymbol{\Lambda}, R, \boldsymbol{Z}_{1}$, and $\boldsymbol{Z}_{2}$ must comply with the positive semidefiniteness of the matrix of transport coefficients,

$\boldsymbol{D}=\left(\begin{array}{ccc}T^{2} \boldsymbol{k} & T \boldsymbol{Z}_{1} & 0 \\ T \boldsymbol{Z}_{1}^{T} & \boldsymbol{\Lambda} & \boldsymbol{Z}_{2} \\ 0 & \boldsymbol{Z}_{2}^{T} & R\end{array}\right) \geq \mathbf{0}$

in order to satisfy all conditions of the thermodynamic framework (see Appendix B for details). The quantity $T$ stands for the generalized temperature defined in Eq. 18 e below.

\section{Time-evolution equations}

In view of the functional derivatives Eq. 42, the Poisson operator Eq. 43, and the friction matrix Eq. 48, and after some mathematical rearrangements, the full set of time-evolution equations assumes the form

$$
\begin{aligned}
\partial_{t} \boldsymbol{u}= & -\nabla \cdot(\boldsymbol{v} \boldsymbol{u})+\nabla \cdot \boldsymbol{\sigma} \\
\partial_{t} \rho= & -\nabla \cdot(\rho \boldsymbol{v}) \\
\partial_{t} \theta= & -\boldsymbol{v} \cdot \nabla \theta+\frac{1}{e_{\mathrm{t}, \theta}}\left[\boldsymbol{\sigma}^{s}:(\nabla \boldsymbol{v})-\nabla \cdot \boldsymbol{j}^{q}-e_{\mathrm{t}, \boldsymbol{c}}:\left(\left.\partial_{t} \boldsymbol{c}\right|_{\mathrm{irr}}\right)\right. \\
& \left.-e_{\mathrm{t}, \lambda}\left(\left.\partial_{t} \lambda\right|_{\mathrm{irr}}\right)\right] \\
\partial_{t} \boldsymbol{c}= & -\boldsymbol{v} \cdot \nabla \boldsymbol{c}+\boldsymbol{\kappa} \cdot \boldsymbol{c}+\boldsymbol{c} \cdot \boldsymbol{\kappa}^{T}+T \boldsymbol{Z}_{1}^{T} \cdot \nabla \frac{1}{T} \\
& +\boldsymbol{\Lambda}:\left(s_{\mathrm{t}, \boldsymbol{c}}-\frac{1}{T} e_{\mathrm{t}, \boldsymbol{c}}\right)+\boldsymbol{Z}_{2}\left(s_{\mathrm{t}, \lambda}-\frac{1}{T} e_{\mathrm{t}, \lambda}\right) \\
\partial_{t} \lambda= & -\boldsymbol{v} \cdot \nabla \lambda+\boldsymbol{\kappa}: \boldsymbol{g} \\
& +\boldsymbol{Z}_{2}^{T}:\left(s_{\mathrm{t}, \boldsymbol{c}}-\frac{1}{T} e_{\mathrm{t}, \boldsymbol{c}}\right)+R\left(s_{\mathrm{t}, \lambda}-\frac{1}{T} e_{\mathrm{t}, \lambda}\right)
\end{aligned}
$$

with the velocity field $\boldsymbol{v}=\boldsymbol{u} / \rho$, the transposed velocity gradient $\kappa=(\nabla v)^{T}$, and where we have defined the stress tensor $\boldsymbol{\sigma}$ and heat flux $\boldsymbol{j}^{q}$, and introduced the abbreviation $T$ as

$\sigma=\sigma^{e}+\sigma^{s}$,

$\boldsymbol{\sigma}^{e}=\left(e_{\mathrm{t}}-\rho e_{\mathrm{t}, \rho}\right) \mathbf{1}+2 \boldsymbol{c} \cdot e_{\mathrm{t}, \boldsymbol{c}}+\boldsymbol{g} e_{\mathrm{t}, \lambda}$,

$\boldsymbol{\sigma}^{s}=-T\left(s_{\mathrm{t}}-\rho s_{\mathrm{t}, \rho}\right) \mathbf{1}-2 T \boldsymbol{c} \cdot s_{\mathrm{t}, \boldsymbol{c}}-\boldsymbol{g} T s_{\mathrm{t}, \lambda}$,

$\boldsymbol{j}^{q}=-\boldsymbol{k} \cdot(\nabla T)+T \mathbf{Z}_{1}:\left(s_{\mathrm{t}, \boldsymbol{c}}-\frac{1}{T} e_{\mathrm{t}, \boldsymbol{c}}\right)$,

$T \equiv e_{\mathrm{t}, \theta} / s_{\mathrm{t}, \theta}$.

In Eqs. 17 and 18, as well as in the remainder of the manuscript, we use the notation $\left.y(\boldsymbol{x})_{, x_{i}} \equiv\left(\partial y / \partial x_{i}\right)\right|_{x_{k \neq i}}$ for partial derivatives. In the expressions for $\sigma_{c}^{e}$ and $\boldsymbol{\sigma}_{c}^{s}$, we have exploited that $e_{\mathrm{t}, \boldsymbol{c}}$ and $s_{\mathrm{t}, \boldsymbol{c}}$ commute with $\boldsymbol{c}$, which is appropriate since $c$ is the only tensor variable. The contributions $\left.\partial_{t} \boldsymbol{c}\right|_{\text {irr }}$ and $\left.\partial_{t} \lambda\right|_{\text {irr }}$ in Eq. $17 \mathrm{c}$ represent the irreversible contributions to the evolution of the conformation tensor $\boldsymbol{c}$ (Eq. 17d) and local structural variable $\lambda$ (Eq. 17e), respectively, i.e., the terms proportional to the transport coefficients $\boldsymbol{\Lambda}, R, \boldsymbol{Z}_{1}$, and $\boldsymbol{Z}_{2}$.

Due to the appearance of $\sigma$ in the momentum balance Eq. $17 \mathrm{a}$, its interpretation as the physical stress tensor is evident. Similarly, the interpretation of $\boldsymbol{j}^{q}$ as the heat flux becomes clear when considering the evolution equation for the total entropy density $s_{\mathrm{t}}$, that can be derived from Eq. 17 by way of the chain rule,

$$
\begin{aligned}
\partial_{t} s_{\mathrm{t}}= & -\nabla \cdot\left(s_{\mathrm{t}} \boldsymbol{v}\right)-\nabla \cdot\left(\frac{1}{T} \boldsymbol{j}^{q}\right) \\
& +\left(\begin{array}{c}
\left(\nabla \frac{1}{T}\right) \\
s_{\mathrm{t}, \boldsymbol{c}}-\frac{e_{\mathrm{t}, \boldsymbol{c}}}{T} \\
s_{\mathrm{t}, \lambda}-\frac{e_{\mathrm{t}, \lambda}}{T}
\end{array}\right) \cdot \boldsymbol{D} \cdot\left(\begin{array}{c}
\left(\nabla \frac{1}{T}\right) \\
s_{\mathrm{t}, \boldsymbol{c}}-\frac{e_{\mathrm{t}, \boldsymbol{c}}}{T} \\
s_{\mathrm{t}, \lambda}-\frac{e_{\mathrm{t}, \lambda}}{T}
\end{array}\right) .
\end{aligned}
$$

The evolution equation for the total energy assumes with

$\partial_{t} e_{\mathrm{t}}=-\nabla \cdot\left(e_{\mathrm{t}} \boldsymbol{v}\right)+(\nabla \boldsymbol{v}): \boldsymbol{\sigma}-\nabla \cdot \boldsymbol{j}^{q}$

the usual form, representing the conservation of the kinetic plus internal energy, $E$.

It is important to note that the driving forces for structural relaxation in Eqs. $17 \mathrm{~d}$ and $17 \mathrm{e}$ and the anisotropic contributions to the stress tensor Eqs. 18a$18 \mathrm{c}$ can only be expressed as the derivative of the Helmholtz free energy density $f_{\mathrm{t}} \equiv e_{\mathrm{t}}-T s_{\mathrm{t}}$, if the temperature $T$ defined in Eq. 18e does not depend on $c$ and $\lambda$, respectively. Whether that condition is fulfilled depends on the choice for the thermal variable $\theta$, as discussed below. In the general case, not the Helmholtz free energy but, rather, the two generators energy and entropy are relevant. 


\section{Discussion}

First, we compare the set of evolution equations (Eq. 17) with the constitutive equations (Eq. 18) with known expressions from literature. To that end, we note that the evolution equation for $\theta$ (Eq. 17c) can be replaced by the equivalent evolution of either $s_{\mathrm{t}}$ (Eq. 19) or $e_{\mathrm{t}}$ (Eq. 20). In the absence of crosscouplings $\left(\boldsymbol{Z}_{1}=\mathbf{0}\right.$ and $\left.\boldsymbol{Z}_{2}=\mathbf{0}\right)$, the evolution equation for $\boldsymbol{c}$ (Eq. 17d) and the expression for the stress tensor specified in Eq. 18 agree with the results in Beris and Edwards (1994), if viscous stresses and nonaffine slip are neglected. The full set of Eqs. 17 and 18 with $\boldsymbol{Z}_{1}=\mathbf{0}$ and $\boldsymbol{Z}_{2}=\mathbf{0}$ is identical to Eqs. 46 and 47 in Dressler et al. (1999), with the major difference that we have included the local scalar variable $\lambda$ for quantification of the energetic effects, in contrast to their procedure with the temperature-dependent overall chain spring constant (see also Eq. 9 above). Furthermore, in contrast to Dressler et al. (1999), we have neglected for simplicity viscous stresses, and external effects on energy and momentum are also not considered. In comparison to Öttinger $(2002,2005)$, there is agreement about the choice of a scalar and tensorial structural variable, and about the corresponding evolution equations in general. However, while our treatment is less broad with respect to the irreversible effects accounted for, we make no restriction on the functional forms of $e_{\mathrm{t}}$ and $s_{\mathrm{t}}$. Particularly, we permit that the conformational contributions to these two functions may depend on the thermal variable $\theta$.

Second, we discuss the meaning of the function $T$ defined in Eq. 18e. For the choice $\theta=e_{\mathrm{t}}$, that definition is formally equivalent to the one for the temperature used in Dressler et al. (1999). On the other hand, if the conformational energy and the conformational entropy are both independent of the thermal variable $\theta$, and with $\theta$ being the local energy density, the definition Eq. 18e reduces to the "local" temperature used by Öttinger (2002, 2005). In both cases, the corresponding temperature enters in the usual way into Fourier's law of heat conduction. While we consider a more general case for the definition of $T$ here, $T$ is still directly related to the driving force for the heat flux Eq. 18d. Furthermore, $T$ also enters into the expression for the stress tensor Eq. 18a-18c. Several terms in the evolution equations (Eq. 17) can be written in a condensed, probably more familiar, form if Eq. 18e allows one to make a variable transformation from $\theta$ to $T$. In this case, one obtains from Eq. 18e the "canonical" consistency relation

$T=\frac{e_{\mathrm{t}, T}(\rho, T, \boldsymbol{c}, \lambda)}{s_{\mathrm{t}, T}(\rho, T, \boldsymbol{c}, \lambda)}$, and $e_{\mathrm{t}, T}$ in Eq. $17 \mathrm{c}$ assumes the meaning of a conformation-dependent heat capacity. Furthermore, with $\theta=T$ and defining the Helmholtz free energy density as $f_{\mathrm{t}}=e_{\mathrm{t}}-T s_{\mathrm{t}}$, one can simplify the driving forces for structural relaxation in Eqs. $17 \mathrm{~d}$ and $17 \mathrm{e}$ to $-f_{\mathrm{t}, c} / T$ and $-f_{\mathrm{t}, \lambda} / T$, respectively, and also the stress tensor expression (Eqs. 18a-18c) assumes a more common form.

What is the relation between the temperature $T$ and the parameter $\beta$ used in the generalized canonical ensemble? Since the GENERIC equations developed above relate to the choice $X_{1}=\boldsymbol{R} \boldsymbol{R}$ and $X_{2}=n$ in the generalized canonical ensemble Eq. 1, one concludes from Eq. 3a that, for $\theta=T$, the parameter $\beta^{-1}$ can be identified with $k_{\mathrm{B}} T$,

$k_{\mathrm{B}} T=\beta^{-1}$.

It is pointed out that this identification holds irrespective of a specific model (e.g., the RIS model as used in the toy example earlier), and it is not restricted to single chain partition functions. While $T$ was related above to the driving force for the heat flux and also entered the stress tensor expression, i.e., it can be interpreted as a "transport" temperature, it becomes clear with Eq. 22 that this temperature also stands for the Lagrange multiplier for the microscopic energy in the setting of a generalized canonical ensemble. Therefore, only now, after establishing the relation between the nonequilibrium thermodynamics treatment and the generalized canonical ensemble, it seems confirmed that using the temperature $T$ as a dynamic variable, as done in Braun and Friedrich (1990), Peters and Baaijens (1997), Wapperom and Hulsen (1998), and Dressler et al. (1999), is indeed admissible. Furthermore, according to Eq. $3 b$, the driving forces for structural relaxation in Eqs. $17 \mathrm{~d}$ and $17 \mathrm{e}$ are given by the Lagrange parameters $\boldsymbol{\alpha}$ and $\mu$, respectively, and the anisotropic contributions to the stress tensor expression (Eq. 18a-18c) can also be rewritten in the form $-k_{\mathrm{B}} T(2 \boldsymbol{c} \cdot \boldsymbol{\alpha}+\boldsymbol{g} \mu)$.

After clarifying the meaning of $T$ as a temperature and establishing its relation to the (microscopic) parameter $\beta$, we are now in the position to discuss the evolution equation of the temperature for flowing polymeric systems. In literature, one usually starts by rewriting Eq. 20 with the aid of the mass balance Eq. $17 \mathrm{~b}$ into an equation for the internal energy per unit mass $\hat{e}_{\mathrm{t}}=e_{\mathrm{t}} / \rho$,

$\rho D_{t} \hat{e}_{\mathrm{t}}=(\nabla \boldsymbol{v}): \boldsymbol{\sigma}-\nabla \cdot \boldsymbol{j}^{q}$,

with the material derivative $D_{t}=\partial_{t}+\boldsymbol{v} \cdot \nabla$ (Braun and Friedrich 1990; Peters and Baaijens 1997; Wapperom and Hulsen 1998; Dressler et al. 1999; Schieber et al. 2004; Ionescu et al. 2008a). Subsequently, as $\hat{e}_{\mathrm{t}}$ is taken 
as a function of the temperature $T$, as well as of the polymer conformation, and the mass density (or the hydrostatic pressure), application of the chain rule to $D_{t} \hat{e}_{t}$ leads to an equation for the temperature of the form

$\rho \hat{e}_{\mathrm{t}, T} D_{t} T=(\nabla \boldsymbol{v}): \sigma-\nabla \cdot \boldsymbol{j}^{q}-\rho \hat{e}_{\mathrm{t}, \boldsymbol{c}}: D_{t} \boldsymbol{c}-\rho \hat{e}_{\mathrm{t}, \rho} D_{t} \rho$.

While the derived results in all of these references are thus equivalent, our equation for $\theta=T$ is most easily compared to the temperature equations in Braun and Friedrich (1990), Peters and Baaijens (1997), Ionescu et al. (2008a), to which Eq. 17c reduces upon neglecting the local variable $\lambda$. A direct consequence of applying the chain rule to $D_{t} \hat{e}_{t}$ is the occurrence of the "heat capacity" $\hat{e}_{\mathrm{t}, T}$, similar to $e_{\mathrm{t}, \theta}$ in Eq. $17 \mathrm{c}$. It is currently debated whether this quantity depends on the conformation of the polymer chains (e.g., Schieber et al. 2004; Ionescu et al. 2008a). We will come back to this issue in the next section when discussing the temperature equation in relation to our microscopic toy model.

The evolution equation Eq. 17c can also be used for other purposes than for deriving a temperature equation. For example, if one is interested in the exchange between local and conformational entropy, it is convenient to choose the local entropy density as thermal variable, $\theta=s_{1}$, and compare the evolution equations Eqs. 17c and 19. To simplify the discussion, we start with the special case that the conformational entropy is independent of $s_{1}$, i.e., $e_{\mathrm{t}, \theta}=T$. In this case, the evolution equations for the local entropy density $s_{1}$ and conformational entropy density $s_{\mathrm{c}}=s_{\mathrm{t}}-s_{\mathrm{l}}$, based on Eq. $17 \mathrm{c}$ with $\theta=s_{1}$ and Eq. 19, can be written in the form

$$
\begin{aligned}
& \partial_{t} s_{1}=-\nabla \cdot\left(s_{1} \boldsymbol{v}\right)+\Sigma_{1}+\Delta_{\mathrm{ex}}^{(s)}-\nabla\left(\frac{\boldsymbol{j}^{q}}{T}\right), \\
& \partial_{t} s_{\mathrm{c}}=-\nabla \cdot\left(s_{\mathrm{c}} \boldsymbol{v}\right)+\Sigma_{\mathrm{t}}-\Sigma_{\mathrm{l}}-\Delta_{\mathrm{ex}}^{(s)}
\end{aligned}
$$

with rate of entropy exchange defined by

$$
\begin{aligned}
\Delta_{\mathrm{ex}}^{(s)}=\frac{1}{T} & {\left[\left(\boldsymbol{\sigma}^{s}+T s_{1} \mathbf{1}\right):(\nabla \boldsymbol{v})-e_{\mathrm{t}, \boldsymbol{c}}:\left(\left.\partial_{t} \boldsymbol{c}\right|_{\mathrm{irr}}\right)\right.} \\
& \left.-e_{\mathrm{t}, \lambda}\left(\left.\partial_{t} \lambda\right|_{\mathrm{irr}}\right)\right],
\end{aligned}
$$

the local entropy production $\Sigma_{1}=\left(\nabla T^{-1}\right) \cdot \boldsymbol{j}^{q}$, and total entropy production $\Sigma_{\mathrm{t}}$ given by the third contribution on the right-hand side of Eq. 19. Note that, for $\boldsymbol{Z}_{1}=\mathbf{0}$ and $\boldsymbol{Z}_{2}=\mathbf{0}$, the quantity $\Sigma_{\mathrm{t}}-\Sigma_{1}$ expresses the positive semidefinite entropy production due to conformational relaxation. The first term in Eq. 24c is reversible in nature and is interpreted as follows.
The flow field distorts the chain conformation, which leads to a decreased conformational entropy. Since the total entropy density is not altered by the reversible dynamics, that loss in conformational entropy must be compensated by the local entropy. This effect is well known in standard classroom experiments on rubber elasticity. The second term in Eq. 24c is irreversible in nature and more subtle since an irreversible exchange also brings about a corresponding entropy production. Due to this joint occurrence of "current" and entropy production, the second term in Eq. 24c must be interpreted as containing both of these contributions, i.e., also including production terms. Assuming $e_{\mathrm{t}, \theta}=T$ for the discussion of entropy transfer is a useful technical and conceptual simplification. When relaxing that assumption, the concept of exchange between local and conformational entropy becomes more subtle, and one may use $s_{\mathrm{t}, s_{1}}-1$ as an expansion parameter by which the scenario for $e_{\mathrm{t}, \theta}=T$ can be extended. Similarly, $\boldsymbol{Z}_{1} \neq \mathbf{0}$ and $\boldsymbol{Z}_{2} \neq \mathbf{0}$ introduce coupling between the local and conformational entropies. However, we shall not enter into these extensions to not distract from the main points of the manuscript.

If, on the other hand, one is interested in the exchange between local and conformational energy, one chooses the local energy density as thermal variable, $\theta=e_{1}$. Given that the conformational energy is independent of $e_{\mathrm{l}}$, i.e., $e_{\mathrm{t}, \theta}=1$, one can proceed in a similar way as in Eq. 24 to identify the rate of exchange between the local energy density $e_{1}$ and conformational energy density $e_{\mathrm{c}}=e_{\mathrm{t}}-e_{1}$. Based on Eqs. $17 \mathrm{c}$ and 20, one obtains

$$
\begin{aligned}
& \partial_{t} e_{\mathrm{l}}=-\nabla \cdot\left(e_{\mathrm{l}} \boldsymbol{v}\right)+\boldsymbol{\sigma}^{s}:(\nabla \boldsymbol{v})+\Delta_{\mathrm{ex}}^{(e)}-\nabla \cdot \boldsymbol{j}^{q}, \\
& \partial_{t} e_{\mathrm{c}}=-\nabla \cdot\left(e_{\mathrm{c}} \boldsymbol{v}\right)+\boldsymbol{\sigma}^{e}:(\nabla \boldsymbol{v})-\Delta_{\mathrm{ex}}^{(e)},
\end{aligned}
$$

with the exchange rate between the local and conformational energy densities

$\Delta_{\mathrm{ex}}^{(e)}=e_{1}(\nabla \cdot \boldsymbol{v})-e_{\mathrm{t}, \boldsymbol{c}}:\left(\left.\partial_{t} \boldsymbol{c}\right|_{\mathrm{irr}}\right)-e_{\mathrm{t}, \lambda}\left(\left.\partial_{t} \lambda\right|_{\mathrm{irr}}\right)$,

that contains an isotropic reversible and an irreversible contribution. Note that the contributions proportional to $(\nabla v)$ in the evolution equations (Eqs. 25a and 25b) express exchange terms between the kinetic energy on the one hand and the local and conformational energies, on the other hand.

\section{Implications of the model}

We now seek to analyze the full set of evolution equations (Eq. 17) with the constitutive equations (Eq. 18) specifically with regard to identifying the effects of the conformational energy. One should note that, for the 
choice $\theta=T$, conformational contributions to the energy density become readily identifiable in $e_{\mathrm{t}}$. Namely, according to Eqs. $2 \mathrm{~b}$ and 31, the total energy density $e_{\mathrm{t}}$ becomes independent of the polymer conformation exactly if the microscopic conformational energy is set to zero, i.e., for $\epsilon=0$. In contrast, if one chose $\theta=e_{\mathrm{t}}$, any explicit dependence of $e_{\mathrm{t}}$ on the conformation would be gone, although that dependence is still present physically in the system. In that spirit, the separation of the stress tensor (Eq. 18a) into energetic and entropic contributions (Eqs. 18b and 18c) is particularly meaningful for $\theta=T$. For all these reasons, we choose $\theta=T=\left(k_{\mathrm{B}} \beta\right)^{-1}$ for the remainder of the paper. Nevertheless, the examination of energetic effects is still complicated since the ramifications of the energy function $e_{\mathrm{t}}(\rho, T, \boldsymbol{c}, \lambda)$ in Eqs. 17 and 18 are multiple, namely, $e_{\mathrm{t}}$ enters in (1) the driving forces for structural relaxation, (2) the stress tensor expression, and (3) several places in the temperature equation.

One possible simplification to the full set of evolution equations consists in looking for spatially homogeneous solutions in a homogeneous time-dependent and incompressible flow, i.e., $\boldsymbol{v}(\boldsymbol{r}, t)=\boldsymbol{\kappa}(t) \cdot \boldsymbol{r}$ with $\operatorname{tr} \kappa=0$. Another possibility is to examine the behavior of the material in the absence of flow. In this latter case, and upon neglecting cross-couplings $\left(\boldsymbol{Z}_{1}=\mathbf{0}\right.$ and $\left.\boldsymbol{Z}_{2}=\mathbf{0}\right)$, the evolution equations (Eq. 17) become

$\mathbf{0}=\nabla \cdot \boldsymbol{\sigma}$

$\partial_{t} \rho=0$,

$e_{\mathrm{t}, T} \partial_{t} T=-\nabla \cdot \boldsymbol{j}^{q}-e_{\mathrm{t}, \boldsymbol{c}}: \partial_{t} \boldsymbol{c}-e_{\mathrm{t}, \lambda} \partial_{t} \lambda$,

$\partial_{t} \boldsymbol{c}=\boldsymbol{\Lambda}:\left(s_{\mathrm{t}, \boldsymbol{c}}-\frac{1}{T} e_{\mathrm{t}, \boldsymbol{c}}\right)$,

$\partial_{t} \lambda=R\left(s_{\mathrm{t}, \lambda}-\frac{1}{T} e_{\mathrm{t}, \lambda}\right)$.

As a special case of these reduced equations, the stationary solutions can be used to study energetic effects. Particularly, with regard to the microscopic toy model introduced earlier, the complete relaxation of $\lambda$ is equivalent to $\mu=0$, according to Eq. $3 \mathrm{~b}$. Following the discussion after Eq. $7 b$, the size of the polymer coil in the relaxed state, as described by $c_{0}(n)$, becomes temperature-dependent if conformational energetic effects are involved, since the equilibrium value of $n$ depends on temperature through Eq. 8. If the polymer coil is fully relaxed also on the large scale, Eqs. $3 \mathrm{~b}$ and 7 a lead to $\boldsymbol{\alpha}=\mathbf{0}$, i.e., $\boldsymbol{c}=c_{0}(n) \mathbf{1}$. Therefore, one can set $Y=0$ in Eq. $8 \mathrm{c}$ to arrive at the temperature dependence of the number of gauche states $n$, and hence of the coil size, $c_{0}(n(T))$. To proceed, we define the local structural variable to be the fraction of occupied gauche states,

$\lambda=n / N$,

where it has again been assumed that the degree of polymerization $N$ is high, $N-2 \simeq N$. Using $\epsilon=$ $2095 \mathrm{~J} \mathrm{~mol}^{-1}$ for polyethylene (Hoeve 1961; Paul et al. 1995), our toy model predicts a change in relative population of gauche states with temperature at $T=420 \mathrm{~K}$ (i.e., in the melt state) in equilibrium as

$d \lambda_{\mathrm{eq}} / d T \simeq 3.5 \times 10^{-4} \mathrm{~K}^{-1}$,

which in turn affects the distribution of the torsion angle $\varphi$. The average $\langle\cos \varphi\rangle$ (Eq. 6b) with $\varphi_{\text {trans }}=0$ and $\varphi_{\text {gauche }} \simeq \pm 2$ changes with temperature as $d\langle\cos \varphi\rangle /$ $d T \simeq-5 \times 10^{-4} \mathrm{~K}^{-1}$. Using the relation Eq. 6a, our toy model thus predicts

$\frac{d \ln C_{\infty}}{d T} \simeq-10^{-3} \mathrm{~K}^{-1}$.

The good agreement of this value with experimental data, $d \ln C_{\infty} / d T \simeq-1.1 \times 10^{-3} \mathrm{~K}^{-1}$ (Hoeve 1961; Boothroyd et al. 1991; Krishnamoorti et al. 2002) overestimates the accuracy of the toy model, given the fact that correlations between torsion angles have been neglected altogether (see Hoeve (1961) for further details).

The reduced equations (Eq. 26) can also be used for studying the material behavior immediately after stopping the flow (see also Schieber et al. 2004). In homogeneous situations and with $e_{\mathrm{t}, c}=\mathbf{0}$ as in our microscopic toy model, the temperature equation assumes the form

$e_{\mathrm{t}, T} \partial_{t} T=-e_{\mathrm{t}, \lambda} \partial_{t} \lambda$,

with, again, $\lambda$ being interpreted as the relative occupation of gauche states defined in Eq. 27. Since the microscopic toy model was intended to highlight the form of the conformational contributions to the energy and entropy and since the single chain approximation is a drastic abstraction, we base our estimate for $e_{\mathrm{t}, T}$ on experimental values rather than on Eq. 4 . For polyethylene, the following value is estimated for $e_{\mathrm{t}, T}=\rho C_{V}$ at equilibrium, where $C_{V}$ is the heat capacity at constant volume per unit mass. Using the relation $\rho\left(C_{p}-C_{V}\right)=T \alpha^{2} / \kappa$ with the heat capacity at constant pressure per unit mass $C_{p}=2202 \mathrm{~J} \mathrm{~kg}^{-1} \mathrm{~K}^{-1}$, the mass density $\rho=780 \mathrm{~kg} \mathrm{~m}^{-3}$, the thermal expansion coefficient $\alpha=7.16 \times 10^{-4} \mathrm{~K}^{-1}$, and the isothermal compressibility $\kappa=10.5 \times 10^{-10} \mathrm{~Pa}^{-1}$, all at $T=420 \mathrm{~K}$ (Orwoll 1996), one obtains $e_{\mathrm{t}, T}=1.5 \times 10^{6} \mathrm{~J} \mathrm{~m}^{-3} \mathrm{~K}^{-1}$. On the other hand, for the microscopic toy model with $e_{\mathrm{t}}=\left(\rho / M_{\mathrm{w}}\right) E^{\mathrm{sc}}$ and $E^{\mathrm{sc}}$ defined in Eq. $4 \mathrm{a}$, one finds 
$e_{\mathrm{t}, \lambda}=\epsilon \rho /\left(M_{\mathrm{w}} / N\right) \simeq 1.2 \times 10^{8} \mathrm{~J} \mathrm{~m}^{-3}$, where we have used $M_{\mathrm{w}} / N=m_{\mathrm{CH}_{2}}=0.014 \mathrm{~kg} \mathrm{~mol}^{-1} .{ }^{1}$ Therefore, the rates of change in the temperature and the relative gauche population are related by

$$
\frac{\partial_{t} \lambda}{\partial_{t} T} \simeq-0.013 \mathrm{~K}^{-1} \text {. }
$$

For example, if, over some small time interval, $\lambda$ relaxes by an amount $\Delta \lambda=0.013$, a temperature change $\Delta T \simeq-1 \mathrm{~K}$ occurs as a result of the effects of conformational energy. The ability of the local variable $\lambda$ to relax by a certain amount depends on how strongly the polymer chain was previously deformed in flow, where the applied flow rate competes with the relaxation effect. In general, note that the relaxation of $\lambda$ is much faster than the relaxation of $\boldsymbol{c}$. It is thus to be expected that, for experimentally measuring the effects of conformational energy by way of Eq. 30, one must be able to capture relatively fast changes in the temperature. Due to the fast relaxation of $\lambda$, the restriction in Eq. 30 to homogeneous situations that preclude thermal conduction to/from the walls of the sample, i.e., to adiabatic conditions, is reasonable if the sample was approximately homogeneous prior to stopping the flow. While detailed flow calculations are required to understand how strongly $\lambda$ can change as a result of certain flow situations, there is an alternative route to at least get a feeling for what $\Delta \lambda=0.013$ stands for. From the relation Eq. 28, one infers that, under equilibrium conditions, a temperature change of the order of $T=37 \mathrm{~K}$ is required to achieve $\Delta \lambda=0.013$.

Finally, we discuss the dependence of the heat capacity $e_{\mathrm{t}, T}$ on the conformation. Dressler et al. (1999), and, subsequently, Ionescu et al. (2008a), have used Eq. 9a, which leads to a heat capacity with a conformation dependent term proportional to the second derivative of the overall chain spring constant, $K^{\prime \prime}(T) \operatorname{tr} c$. In our toy model, in contrast, $e_{\mathrm{t}}$ is completely independent of $\boldsymbol{c}$, and the term that contains the local structure through $\lambda$ is athermal. As a result, in our case, the heat capacity $e_{\mathrm{t}, T}$ is not affected by the chain conformation, neither through $\boldsymbol{c}$, nor through $\lambda$. Due to the absence of such couplings, the heat capacity is rather insensitive to flow. If this is found to be in conflict with experimental

\footnotetext{
${ }^{1}$ Note that experimental values for the heat capacity include, in addition to the change in the vibrational behavior described by the derivative $e_{\mathrm{t}, T}$ at constant $\lambda$, also the change of the trans/gauche population with respect to temperature. With the above numbers, one obtains for that contribution $\rho C_{V}-e_{\mathrm{t}, T}=$ $e_{\mathrm{t}, \lambda} d \lambda_{\mathrm{eq}} / d T \simeq 4.2 \times 10^{4} \mathrm{~J} \mathrm{~m}^{-3} \mathrm{~K}^{-1}$. As this is less than $3 \%$ of the total value, this correction has been neglected in the estimation of $\rho C_{V}$.
}

findings, one concludes that the weak dependence of the local Helmholtz free energy $F_{\text {loc }}$ on $n$ discussed in Eq. 40 is too drastic a simplification, and must, hence, be relaxed. Nevertheless, a formal connection to Dressler et al. (1999) and Ionescu et al. (2008a) can be made by completely relaxing the fast local variable $n$. Doing so leads to the $c$ dependence of the energy Eq. 10, which in turn amounts to a heat capacity that depends on the conformation tensor. However, as pointed out previously, we emphasize again the need to explicitly use the local structural variable as an independent quantity, since the degrees of freedom that carry conformational energy are local and have different dynamics compared to the conformation tensor.

\section{Summary and perspectives}

A suitable and manageable treatment of energy elastic effects in flowing polymeric systems requires a thoughtful discussion of the set of independent variables, namely due to the significant departure from equilibrium conditions. In this paper, we advocate the use of a local structural variable for capturing the energetic contributions in a direct manner, in addition to a tensorial variable that accounts for the slow, large scale features. By calculating the generalized canonical partition function for a simple microscopic toy model, the microscopic understanding of the energetics was translated into two out-of-equilibrium thermodynamic functions, the energy density $e_{\mathrm{t}}$ and the entropy density $s_{\mathrm{t}}$. The latter then enter into the formulation of a set of thermodynamically admissible evolution equations. In the most general case, the driving force for structural relaxation and the conformational contributions to the stress tensor have been found not to be proportional to the derivatives of the Helmholtz free energy density; rather, combinations of the derivatives of the energy and entropy densities are the more fundamental quantities. Furthermore, it has been demonstrated that the common use of the temperature as a dynamic variable is indeed possible for these systems. In particular, it has been shown that the temperature used in the definition of the heat flux is directly related to the Lagrange multiplier of the microscopic energy in the generalized canonical partition function. Specifically, with regard to the microscopic toy model, the following points are important: First, upon complete relaxation of the local structural variable, the energy of the toy model has been shown to reduce to known expressions in literature where only a conformation tensor is used. Second, the prediction about the change in the size of the polymer coil with temperature, which 
is due to energetic effects, for polyethylene is in agreement with experimental data. Third, the heat capacity occurring naturally in the temperature equation does not depend on the polymer conformation. Fourth, it is predicted for polyethylene that, after stopping the flow, the rate of relaxation $\dot{\lambda}$ of the relative gauche population, $\lambda=n / N$, leads to a temperature change $\dot{T}$ caused by the conformational energetic effects of the order $\dot{T} \simeq-\dot{\lambda} / 0.013 \mathrm{~K}^{-1}$, under adiabatic conditions. In order to discuss the absolute magnitude of the expected temperature changes during relaxation, further studies are needed to understand how strongly $\lambda$ can depart from its equilibrium value as a result of certain flow situations. Finally, based on the general evolution equations, the exchange between local and conformational energy (and entropy, respectively) upon deformation has also been addressed.

The range of applicability of the GENERIC model developed above goes far beyond the microscopic toy model in several respects. The latter was specific in the sense that the local structural variable was chosen as the number of gauche states $\lambda=n$ and the tensorial variable as $\boldsymbol{c}=\langle\boldsymbol{R} \boldsymbol{R}\rangle$ with $\boldsymbol{R}$ the end-to-end vector. However, there already exist several models in literature that use a scalar and a tensorial variable to describe polymeric liquids. For example, the pompon model for melts of multiply branched polymers (McLeish and Larson 1998; Öttinger 2001) is included in the above GENERIC model (Öttinger 2002, 2005; Soulages et al. 2006), and also some versions of the reptation model (Ianniruberto and Marrucci 2001; Marrucci and Ianniruberto 2003) fall into the same class. In both of these examples, the scalar structural variable represents local chain (segment) stretch that relaxes substantially faster than the tensorial variable. Therefore, the above general set of evolution equations push the door wide open for studying energy elastic effects also in branched and entangled polymer melts. The relevance of the local chain stretch for describing energetic effects is particularly clear in the case of polyethylene, where the number of gauche states is intimately related to the persistence length. In this respect, it is helpful to extend Monte Carlo simulations used by Mavrantzas and Theodorou (1998), Mavrantzas and Öttinger (2002), and Ionescu et al. (2008b) as to configurationally bias rather local structural variables, in contrast or in addition to the conformation tensor. Significant steps in this direction have recently been made by the so-called semigrand canonical Monte Carlo method of Bernardin and Rutledge (2007a, b).

In the toy model, conformational energetic effects entered only through the intrachain torsional degrees of freedom. Certainly, there are other conformational energies to be considered for realistic polymers, e.g., nonbonded intrachain and interchain Lennard-Jones interactions, as discussed in detail by Gao and Weiner (1992, 1994) and Ionescu et al. (2008b). As far as their representation in the energy and entropy densities $e_{\mathrm{t}}$ and $s_{\mathrm{t}}$ is concerned, it seems clear that density (or pressure) effects are important. As for the conformation of the polymer chains, it is to be expected that local structural features are more relevant than long-range ones. As an extreme example, crystallizable polymers hint in this direction since local orientation and packing effects are much more relevant than the large-scale conformation tensor for the formation of nuclei and crystallization (Ko et al. 2004; Van Meerveld et al. 2004). Therefore, again, one is led to use a local structural variable, $\lambda$, for an adequate description of such nonbonded energetic effects. In this regard, it is evident that, with the GENERIC model presented here, a large and rich class of models can now be considered in a setting that is appropriate for beyond equilibrium situations.

\section{Appendix}

A Toy model: calculation of the energy and the entropy for a single chain

For describing the polymer chain in situations out of equilibrium, we introduce $\alpha$ and $\mu$ as the Lagrange multipliers to the structural variables $X_{1}=\boldsymbol{R} \boldsymbol{R}$ and $X_{2}=$ $n$, respectively, and calculate the generalized canonical partition function $Z(\beta, \alpha, \mu)$. If $\boldsymbol{R}$ and $\boldsymbol{Q}$ denote the end-to-end vectors of a specific RIS conformation $j$ with and without vibrations $\boldsymbol{r}$, respectively, i.e., $\boldsymbol{R}=$ $\boldsymbol{Q}+\boldsymbol{r}$, and $z$ the Cartesian microscopic positions of the $N$ atoms relative to the RIS conformation, the partition function Eq. 1 can be written in the form

$$
\begin{aligned}
Z(\beta, \boldsymbol{\alpha}, \mu)= & \sum_{n=0}^{N-2} e^{-\mu n-\beta H_{n}} \\
& \times \sum_{j \in J_{n}} \int_{\mathcal{C}_{j}} e^{-\beta \hat{H}(z ; \mathrm{RIS})-\boldsymbol{\alpha}:(\boldsymbol{Q}+\boldsymbol{r})(\boldsymbol{Q}+\boldsymbol{r})} d z,
\end{aligned}
$$

with $J_{n}$ the set of RIS states that consists of $n$ gauche states, and $\mathcal{C}_{j}$ is the domain in configuration space in the vicinity of the RIS state $j$. Note that vibrations are better characterized with $z$ interpreted as Cartesian deviations from the RIS state, since even small variations in bond angles and torsion angles would lead to (unphysically) large Cartesian displacements of the atoms for long chains. The right-hand side in Eq. 31 
illustrates the decomposition of phase space into (1) a sum over the number of gauche states, which, in turn, is refined to (2) a summation over all RIS conformations with $n$ gauche states, followed by (3) the local vibrations around each RIS state [see also Öttinger (2007) for a similar splitting in the context of glasses]. The energy $H$ is decomposed into the energy associated with the number of gauche states, $H_{n}=\epsilon n$, and a term that accounts for the local vibrations around the RIS conformation, $\hat{H}$ with $\hat{H}(z=0)=0$. For rather stiff interaction potentials, the integration domain $\mathcal{C}_{j}$ can be extended to $\mathbb{R}^{3 N}$ without changing the integral significantly. Therefore, one can interchange the summation $\sum_{j \in J_{n}}$ and the integration $\int \ldots d z$.

To proceed, we make the plausible assumption that $\hat{H}(z$; RIS) depends on local aspects of the RIS conformation, and not on the corresponding end-to-end vector that is a large-scale quantity. Therefore, the first and second terms in the second exponential in Eq. 31 feel different aspects of the RIS conformation. For large $N, \boldsymbol{Q}$ is Gaussian distributed with zero mean and a variance that depends not only on $N$ but also on the number of gauche states $n$, i.e., $\langle\boldsymbol{Q}\rangle=\mathbf{0}$ and $\langle\boldsymbol{Q} \boldsymbol{Q}\rangle=$ $c_{0}(n ; N) \mathbf{1}$. With the assumption that the energy $\hat{H}$ and the $\boldsymbol{\alpha}$-term are mutually uncorrelated and that the local fluctuations $z$ are primarily relevant for the energy $\hat{H}$ but not for $\boldsymbol{R}$, i.e., $\boldsymbol{R} \rightarrow \boldsymbol{Q}$ by virtue of stiff interaction potentials, one can treat the $\beta$ and $\alpha$ terms in the integral in Eq. 31 separately. In particular, $e^{-\boldsymbol{\alpha}: \boldsymbol{Q} \boldsymbol{Q}}$ for given $j \in J_{n}$, can be replaced by its average with respect to the Gaussian distribution function for $\boldsymbol{Q}$, i.e., by $\sqrt{\operatorname{det}\left(\boldsymbol{c} / c_{0}\right)}$, where we have defined the $n$-dependent quantity

$\boldsymbol{c}^{-1}=2 \boldsymbol{\alpha}+\frac{1}{c_{0}(n ; N)} \mathbf{1}$.

The partition function Eq. 31 can be rewritten further by introducing the definitions for the $n$ contribution to the conformational entropy and the local Helmholtz free energy,

$S_{\text {conf }}(n)=k_{\mathrm{B}} \ln \mathcal{N}(n)$,

$F_{\text {loc }}(n, \beta)=-\beta^{-1} \ln \left\{\frac{1}{\mathcal{N}(n)} \sum_{j \in J_{n}} Z_{j}\right\}$,

with $\mathcal{N}$ the number of possible RIS conformations for given $n$, and $Z_{j}$ the partition function accounting for the local vibrations around the RIS state $j$,

$$
\begin{aligned}
& \mathcal{N}(n)=2^{n}\left(\begin{array}{c}
N-2 \\
n
\end{array}\right), \\
& Z_{j}=\int e^{-\beta \hat{H}(z ; \mathrm{RIS})} d z .
\end{aligned}
$$

Since the summand in the partition function Eq. 31 is strongly peaked with respect to $n$, a saddle-point approximation can be used, leading to the condition for $n=n(\beta, \boldsymbol{\alpha}, \mu)$,

$\mu=-\beta \epsilon+\frac{\partial\left(S_{\text {conf }} / k_{\mathrm{B}}\right)}{\partial n}-\beta \frac{\partial F_{\mathrm{loc}}}{\partial n}-\left(\boldsymbol{c} / c_{0}\right): \alpha \frac{\partial c_{0}}{\partial n}$.

The partition function then assumes the form

$Z(\beta, \boldsymbol{\alpha}, \mu)=e^{-\mu n-\beta H_{n}+\frac{S_{\text {conf }}}{k_{\mathrm{B}}}-\beta F_{\mathrm{loc}}-\frac{1}{2} \ln \operatorname{det}\left(c_{0} c^{-1}\right)}$,

with $H_{n}=n \epsilon$, and $S_{\text {conf }}(n)$ and $F_{\text {loc }}(n, \beta)$ given by Eqs. 33 and 34, respectively.

According to Eq. 2 and using the condition Eq. 37, one arrives at the main result of this toy model, namely the expressions for the energy and entropy, and the averages of the conformational variables,

$$
\begin{aligned}
& E=\left(F_{\mathrm{loc}}-\left.T \frac{\partial F_{\mathrm{loc}}}{\partial T}\right|_{n}\right)+\epsilon n, \\
& S=-\left.\frac{\partial F_{\mathrm{loc}}}{\partial T}\right|_{n}+S_{\mathrm{conf}}+\frac{k_{\mathrm{B}}}{2}\left[\left(3-\frac{\operatorname{tr}(\boldsymbol{c})}{c_{0}}\right)-\ln \operatorname{det}\left(c_{0} c^{-1}\right)\right],
\end{aligned}
$$

$\langle\boldsymbol{R} \boldsymbol{R}\rangle=\boldsymbol{c}$,

$\langle n\rangle=n$.

While the expressions on the right-hand side of Eq. 39 are expressed in terms of the variables $(\mu, \beta, \boldsymbol{\alpha})$, one can eliminate $(\mu, \boldsymbol{\alpha})$ in favor of $(n, \boldsymbol{c})$ and express $E$ and $S$ in terms of the variables $(n, \beta, c)$.

In order to obtain fully explicit expressions for the energy and entropy, the local Helmholtz free energy $F_{\text {loc }}$ defined by Eqs. 34-36 must be specified. Since we concentrate on the effects of conformation, we shall not delve into this point. We just mention that the most promising route in this respect seems to be the use of Monte Carlo simulations of (united) atoms in an ensemble with the control parameters $\beta$ and $n$, for a single chain. The influence of the number $n$ of gauche states on $F_{\text {loc }}$ can be examined by looking at the derivative $\partial F_{\text {loc }}(\beta, n) / \partial n$, which can be approximated by

$$
\beta \frac{\partial F_{\mathrm{loc}}(\beta, n)}{\partial n} \simeq \frac{\sum_{j \in J_{n}} \int e^{-\beta \hat{H}(z ; \mathrm{RIS})}\left(e^{-\beta \Delta \hat{H}}-1\right) d z}{\sum_{j \in J_{n}} \int e^{-\beta \hat{H}(z ; \mathrm{RIS})} d z},
$$

where $\Delta \hat{H}$ stands for the energy difference arising in $\hat{H}$ as a result of flipping one bond along the chain from the trans state into a gauche state. If that energy difference is significantly smaller than the thermal energy $k_{\mathrm{B}} T$ for all values of $z$ that contribute to the integral in Eq. 40, then the local energy and entropy can be considered 
effectively independent of $n$. That condition is fulfilled if the form of the atomistic potentials around $z=0$ is insensitive to $n$.

In order to discuss whether the ensemble chosen above at constant $(\beta, \boldsymbol{\alpha}, \mu)$ is equivalent to other ensembles that control $H, \boldsymbol{R} \boldsymbol{R}$, and $n$, respectively, one must examine if the fluctuations in the above ensemble are small,

$\left|\frac{\partial^{2} \ln Z}{\partial \gamma_{i} \partial \gamma_{k}}\right| \ll\left|\frac{\partial \ln Z}{\partial \gamma_{i}}\right|\left|\frac{\partial \ln Z}{\partial \gamma_{k}}\right|$,

where $\gamma_{i}$ and $\gamma_{k}$ are any of the Lagrange parameters $(\beta, \boldsymbol{\alpha}, \mu)$. Except if both $\gamma_{i}$ and $\gamma_{k}$ represent elements of $\alpha$, the conditions Eq. 41 can indeed be shown to hold for large $N$, i.e., fluctuations are small. For the conformation tensor $\boldsymbol{c}$, however, the fluctuations are not small; these fluctuations are given by the Gaussian nature of the distribution of $\boldsymbol{R}$. A generalized microcanonical distribution for the conformation, for example, would destroy long fluctuations expressing Gaussian behavior. The generalized canonical distribution based on the dyadic $\boldsymbol{R} \boldsymbol{R}$, on the other hand, nicely represents the underlying Gaussian statistics.

B Formulation of the evolution equations in the GENERIC framework

\section{Functional derivatives}

The functional derivatives of Eq. 14, that drive the reversible and irreversible contributions in the evolution equation (Eq. 11), are given by

$$
\frac{\delta E}{\delta \boldsymbol{x}}=\left(\begin{array}{c}
\boldsymbol{v} \\
-\frac{1}{2} \boldsymbol{v}^{2}+e_{\mathrm{t}, \rho} \\
e_{\mathrm{t}, \theta} \\
e_{\mathrm{t}, \boldsymbol{c}} \\
e_{\mathrm{t}, \lambda}
\end{array}\right), \quad \frac{\delta S}{\delta \boldsymbol{x}}=\left(\begin{array}{c}
\mathbf{0} \\
s_{\mathrm{t}, \rho} \\
s_{\mathrm{t}, \theta} \\
s_{\mathrm{t}, \boldsymbol{c}} \\
s_{\mathrm{t}, \lambda}
\end{array}\right)
$$

with the velocity field defined by $\boldsymbol{v}=\boldsymbol{u} / \rho$. In Eq. 42, as well as in the remainder of the manuscript, we use the notation $\left.y(\boldsymbol{x})_{, x_{i}} \equiv\left(\partial y / \partial x_{i}\right)\right|_{x_{k \neq i}}$ for partial derivatives.

\section{Reversible dynamics}

The reversible contributions to the full evolution equations of the variables $\boldsymbol{x}$ are of purely kinematic origin, and, hence, directly related to the velocity field. For our specific choice of variables and in view of the functional derivatives Eq. 42, one arrives at the following expres- sion for the Poisson operator (see also Dressler et al. 1999; Öttinger 2002)

$\boldsymbol{L}=-\left(\begin{array}{ccccc}\nabla_{\gamma} u_{\alpha}+u_{\gamma} \nabla_{\alpha} & \rho \nabla_{\alpha} & L_{\alpha}^{(u \theta)} & L_{\alpha \gamma \varepsilon}^{(u c)} & L_{\alpha}^{(u \lambda)} \\ \nabla_{\gamma} \rho & 0 & 0 & 0 & 0 \\ L_{\gamma}^{(\theta u)} & 0 & 0 & 0 & 0 \\ L_{\alpha \beta \gamma}^{(c u)} & 0 & 0 & 0 & 0 \\ L_{\gamma}^{(\lambda u)} & 0 & 0 & 0 & 0\end{array}\right)$,

with

$$
\begin{aligned}
& L_{\alpha \gamma \varepsilon}^{(u c)} \equiv-\left(\nabla_{\alpha} c_{\gamma \varepsilon}\right)-\nabla_{\mu} c_{\mu \gamma} \delta_{\alpha \varepsilon}-\nabla_{\mu} c_{\mu \varepsilon} \delta_{\alpha \gamma}, \\
& L_{\alpha \beta \gamma}^{(c u)} \equiv\left(\nabla_{\gamma} c_{\alpha \beta}\right)-c_{\alpha \mu} \nabla_{\mu} \delta_{\beta \gamma}-c_{\beta \mu} \nabla_{\mu} \delta_{\alpha \gamma}, \\
& L_{\alpha}^{(u \lambda)}=-\left(\nabla_{\alpha} \lambda\right)-\nabla_{\mu} g_{\alpha \mu}, \\
& L_{\gamma}^{(\lambda u)}=\left(\nabla_{\gamma} \lambda\right)-g_{\gamma \mu} \nabla_{\mu}, \\
& L_{\alpha}^{(u \theta)}=-\rho \nabla_{\alpha} \frac{s_{\mathrm{t}, \rho}}{s_{\mathrm{t}, \theta}}+s_{\mathrm{t}} \nabla_{\alpha} \frac{1}{s_{\mathrm{t}, \theta}} \\
& -L_{\alpha \gamma \varepsilon}^{(u c)} \frac{s_{\mathrm{t}, c_{\gamma \varepsilon}}}{s_{\mathrm{t}, \theta}}-L_{\alpha}^{(u \lambda)} \frac{s_{\mathrm{t}, \lambda}}{s_{\mathrm{t}, \theta}}, \\
& L_{\gamma}^{(\theta u)}=-\frac{s_{\mathrm{t}, \rho}}{s_{\mathrm{t}, \theta}} \nabla_{\gamma} \rho+\frac{1}{s_{\mathrm{t}, \theta}} \nabla_{\gamma} s_{\mathrm{t}} \\
& -\frac{s_{\mathrm{t}, c_{\alpha \beta}}}{s_{\mathrm{t}, \theta}} L_{\alpha \beta \gamma}^{(c u)}-\frac{s_{\mathrm{t}, \lambda}}{s_{\mathrm{t}, \theta}} L_{\gamma}^{(\lambda u)} \text {. }
\end{aligned}
$$

Subscripts $(\alpha, \beta)$ and $(\gamma, \varepsilon)$ imply contraction with tensors multiplied from the left and from the right, respectively. All derivative operators act on everything to their right, also on functions multiplied to the right of $\boldsymbol{L}$, except when placed inside of parentheses (...). The element $L^{(c u)}$ dictates the convection of the tensor $\boldsymbol{c}$, i.e., upper convected Maxwell behavior. The convection of $\lambda$, determined by $L^{(\lambda u)}$, is coupled to $\boldsymbol{c}$ through $\boldsymbol{g}$, that is a function of $\lambda$ and $\boldsymbol{c}$. Since $\lambda$ is a scalar, such coupling is required to describe, for example, orientationdependent local chain stretching upon the application of flow. The complicated expression for $L^{(u \theta)}$ is given by the degeneracy requirement Eq. 12a, which in turn fixes $L^{(\theta u)}$ by the antisymmetry condition. In the middle term of $L^{(u \theta)}$, the prefactor $s_{\mathrm{t}}$ is chosen for dimensional reasons and in view of the evolution equation for the total entropy density, $s_{\mathrm{t}}$, see Eq. 19.

The Poisson operator Eq. 43 is antisymmetric and satisfies the degeneracy requirement by construction. It can also be shown that the Jacobi identity is satisfied under the following condition (Öttinger 2002). Using the representation theorem of tensor functions, the most general form of $\boldsymbol{g}$ is given by

$\boldsymbol{g}=g_{1} \boldsymbol{c}+g_{2} \mathbf{1}+g_{3} \boldsymbol{c}^{-1}$, 
with the scalar coefficient functions

$g_{j}=g_{j}\left(\lambda, I_{1}, I_{2}, I_{3}\right)$

depending on $\lambda$ and the three independent invariants

$I_{1}=\operatorname{tr} \boldsymbol{c}$,

$I_{2}=\ln \operatorname{det} \boldsymbol{c}$,

$I_{3}=-\operatorname{tr}\left(c^{-1}\right)$.

It can be shown that the Jacobi identity is satisfied under the conditions

$g_{i} \frac{\partial g_{k}}{\partial \lambda}-g_{k} \frac{\partial g_{i}}{\partial \lambda}=2\left(\frac{\partial g_{i}}{\partial I_{k}}-\frac{\partial g_{k}}{\partial I_{i}}\right)$

for all pairs $(i, k)$. Applying $\boldsymbol{L}$ to $\delta E / \delta \boldsymbol{x}$ leads to the reversible contributions to the evolution equations of $\boldsymbol{x}$, which are summarized in Eq. 17.

\section{Irreversible dynamics}

We discuss the irreversible effects arising as a result of anisotropic heat conduction, and relaxation of the conformational variables. For simplicity, anisotropic viscous flow and slip effects on the conformation tensor, as occurring in Schowalter derivatives, are not included. In view of the GENERIC formalism (Eq. 11), all these irreversible effects come into play through the metric matrix $\boldsymbol{M}$. In order to present the procedure in a transparent form, we split the metric matrix according to Edwards (1998)

$$
\boldsymbol{M}=\boldsymbol{C} \cdot \boldsymbol{D} \cdot \boldsymbol{C}^{T}
$$

The special construction Eq. 48 ensures that $\boldsymbol{M}$ is symmetric and positive semidefinite if $\boldsymbol{D}$ has these properties. The splitting Eq. 48 is done in such a way that the degeneracy requirement Eq. $12 \mathrm{~b}$ is satisfied by the condition $\boldsymbol{C}^{T} \cdot \delta E / \delta \boldsymbol{x}=\mathbf{0}$, and the quantity $\boldsymbol{C}^{T} \cdot \delta S / \delta \boldsymbol{x}$ can be interpreted as the driving force for the irreversible processes. Since both viscous flow and slip effects are neglected, we expect the thermodynamic driving forces to be independent of the velocity field. In order to achieve that, we choose the $\boldsymbol{u}$ - and $\rho$-columns of $\boldsymbol{C}^{T}$ to vanish, since otherwise, the degeneracy condition would bring about spurious velocity terms into the driving forces. Due to the restriction to heat conduction and structural relaxation, only three thermodynamic driving forces are relevant. For all these reasons, we propose the following form of the operator $\boldsymbol{C}^{T}$, and hence, of $\boldsymbol{C}$,

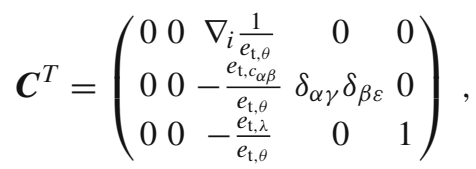

$\boldsymbol{C}=\left(\begin{array}{ccc}0 & 0 & 0 \\ 0 & 0 & 0 \\ -\frac{1}{e_{\mathrm{t}, \theta}} \nabla_{k} & -\frac{e_{\mathrm{t}, c_{\gamma \varepsilon}}}{e_{\mathrm{t}, \theta}} & -\frac{e_{\mathrm{t}, \lambda}}{e_{\mathrm{t}, \theta}} \\ 0 & \delta_{\alpha \gamma} \delta_{\beta \varepsilon} & 0 \\ 0 & 0 & 1\end{array}\right)$.

The three rows in Eq. 49a are unique in that they lead to sensible driving forces for heat conduction and conformational relaxation, that are given by

$$
\boldsymbol{C}^{T} \cdot \frac{\delta S}{\delta \boldsymbol{x}}=\left(\begin{array}{c}
\nabla \frac{1}{T} \\
s_{\mathrm{t}, \boldsymbol{c}}-\frac{1}{T} e_{\mathrm{t}, \boldsymbol{c}} \\
s_{\mathrm{t}, \lambda}-\frac{1}{T} e_{\mathrm{t}, \lambda}
\end{array}\right),
$$

where we have used the abbreviation $T=e_{\mathrm{t}, \theta} / s_{\mathrm{t}, \theta}$. For the matrix of transport coefficients, we write

$$
\boldsymbol{D}=\left(\begin{array}{ccc}
T^{2} \boldsymbol{k} & T \boldsymbol{Z}_{1} & 0 \\
T \boldsymbol{Z}_{1}^{T} & \boldsymbol{\Lambda} & \boldsymbol{Z}_{2} \\
0 & \boldsymbol{Z}_{2}^{T} & R
\end{array}\right) \geq \mathbf{0},
$$

where the second rank tensor $\boldsymbol{k}$ takes the meaning of the anisotropic thermal conductivity and the fourth rank tensor $\Lambda$ and $R$ describe the relaxation of the conformation tensor $\boldsymbol{c}$ and scalar variable $\lambda$, respectively. The off-diagonal elements are inserted to account for possible cross-effects in the force-flux relations. Due to the disparities in length scales, it is assumed here that the local structural features captured by $\lambda$ are not affected by temperature gradients, and hence, the corresponding off-diagonal element is zero. Any ansatz for the quantities $\boldsymbol{k}, \boldsymbol{\Lambda}, R, \boldsymbol{Z}_{1}$, and $\boldsymbol{Z}_{2}$ must comply with the positive semidefiniteness of $\boldsymbol{D}$. Applying $\boldsymbol{M}$ to $\delta S / \delta \boldsymbol{x}$ leads to the irreversible contributions to the evolution equations of $\boldsymbol{x}$.

\section{References}

Astarita G (1974) Thermodynamics of dissipative materials with entropic elasticity. Polym Eng Sci 14:730-733

Astarita G, Sarti GC (1976) The dissipative mechanism in flowing polymers: theory and experiments. J Non-Newton Fluid Mech 1:39-50

Baig C, Mavrantzas VG (2007) Thermodynamically guided nonequilibrium Monte Carlo method for generating realistic shear flows in polymeric systems. Phys Rev Lett 99:257801

Beris AN, Edwards BJ (1994) Thermodynamics of flowing systems with internal microstructure. Oxford University Press, Oxford 
Bernardin III FE, Rutledge GC (2007a) Semi-grand canonical Monte Carlo (SGMC) simulations to interpret experimental data on processed polymer melts and glasses. Macromolecules 40:4691-4702

Bernardin III FE, Rutledge GC (2007b) Simulation of mechanical properties of oriented glassy polystyrene. Polymer 48:7211-7220

Bird RB, Armstrong RC, Hassager O, Curtiss CF (1977) Dynamics of polymeric liquids. Kinetic theory, vol 2. Wiley, New York

Boothroyd AT, Rennie AR, Boothroyd CB (1991) Direct measurement of the temperature dependence of the unperturbed dimensions of a polymer. Europhys Lett 15:715-719

Braun H, Friedrich C (1990) Dissipative behavior of viscoelastic fluids derived from rheological constitutive equations. J Non-Newton Fluid Mech 38:81-91

Callen HB (1985) Thermodynamics and an introduction to thermostatistics. Wiley, New York

Casas-Vázquez J, Jou D (2003) Temperature in non-equilibrium states: a review of open problems and current proposals. Rep Prog Phys 66:1937-2023

Dressler M, Edwards BJ, Öttinger HC (1999) Macroscopic thermodynamics of flowing polymeric liquids. Rheol Acta 38:117-136

Edwards BJ (1998) An analysis of single and double generator thermodynamic formalisms for the macroscopic description of complex fluids. J Non-equilib Thermodyn 23: 301-333

Edwards BJ, Öttinger HC (1997) Time-structure invariance criteria for closure approximations. Phys Rev E 56:4097-4103

Flory PJ (1988) Statistical mechanics of chain molecules. Hanser, Munich

Gao J, Weiner JH (1992) Stress relaxation in a polymer melt of freely-rotating chains. J Chem Phys 97:8698-8704

Gao J, Weiner JH (1994) Simulation of viscoelasticity in polymer melts: effect of torsional potential. Model Simul Mater Sci Eng 2:755-766

Grmela M (1998) Letter to the editor: comment on "thermodynamics of viscoelastic fluids: the temperature equation [J. Rheol. 42, 999 (1998)]". J Rheol 42:1565-1567

Grmela M, Öttinger HC (1997) Dynamics and thermodynamics of complex fluids. I. Development of a general formalism. Phys Rev E 56:6620-6632

Hoeve CAJ (1961) Unperturbed mean-square end-to-end distance of polyethylene. J Chem Phys 35:1266-1267

Ianniruberto G, Marrucci G (2001) A simple constitutive equation for entangled polymers with chain stretch. J Rheol 45:1305-1318

Ionescu TC, Edwards BJ, Keffer DJ (2008a) Energetic and entropic elasticity of nonisothermal flowing polymers: experiment, theory, and simulation. J Rheol 52:105-140

Ionescu TC, Mavrantzas VG, Keffer DJ, Edwards BJ (2008b) Atomistic simulation of energetic and entropic elasticity in short-chain polyethylenes. J Rheol 52:567-589

In 't Veld PJ, Hütter M, Rutledge GC (2006) Temperaturedependent thermal and elastic properties of the interlamellar phase of semicrystalline polyethylene by molecular simulation. Macromolecules 39:439-447

Ko MJ, Waheed N, Lavine MS, Rutledge GC (2004) Characterization of polyethylene crystallization from an oriented melt by molecular dynamics simulation. J Chem Phys 121: 2823-2832

Krishnamoorti R, Graessley WW, Zirkel A, Richter D, Hadjichristidis N, Fetters LJ, Lohse DJ (2002) Melt-state polymer chain dimensions as a function of temperature. J Polym Sci Polym Phys 40:1768-1776
Landau LD, Lifshitz EM (1980) Course of theoretical physics (vols 5 \& 9) (3rd ed). Pergamon, Oxford

Larson RG (1988) Constitutive equations for polymer melts and solutions. Butterworth, Stoneham

Marrucci G, Ianniruberto G (2003) Flow-induced orientation and stretching of entangled polymers. Philos Trans R Soc A 361:677-688

Mattice WL, Suter UW (1994) Conformational theory of large molecules: the rotational isomeric state model in macromolecular systems. New York, Wiley

Mavrantzas VG, Öttinger HC (2002) Atomistic Monte Carlo simulations of polymer melt elasticity: their nonequilibrium thermodynamics GENERIC formulation in a generalized canonical ensemble. Macromolecules 35:960-975

Mavrantzas VG, Theodorou DN (1998) Atomistic simulation of polymer melt elasticity: calculation of the free energy of an oriented polymer melt. Macromolecules 31:6310-6332

McLeish TCB, Larson RG (1998) Molecular constitutive equations for a class of branched polymers: the pom-pom polymer. J Rheol 42:81-110

Orwoll RA (1996) Densities, coefficients of thermal expansion, and compressibilities of amorphous polymers. In: Mark JE (ed) Physical properties of polymers handbook, chap. 7. American Institute of Physics, Woodbury

Öttinger HC (1999) A thermodynamically admissible reptation model for fast flows of entangled polymers. J Rheol 43: $1461-1493$

Öttinger HC (2001) Thermodynamic admissibility of the pompon model for branched polymers. Rheol Acta 40:317-321

Öttinger HC (2002) Modeling complex fluids with a tensor and a scalar as structural variables. Rev Mex Fis 48(Suppl 1): 220-229

Öttinger HC (2005) Beyond equilibrium thermodynamics. Wiley, Hobroken

Öttinger HC (2007) Nonequilibrium thermodynamics of glasses. Phys Rev E 74:011113(1-25)

Öttinger HC, Grmela M (1997) Dynamics and thermodynamics of complex fluids. II. Illustrations of a general formalism. Phys Rev E 56:6633-6655

Paul W, Yoon DY, Smith GD (1995) An optimized united atom model for simulations of polymethylene melts. J Chem Phys 103:1702-1709

Peters GWM, Baaijens FPT (1997) Modelling of non-isothermal viscoelastic flows. J Non-Newton Fluid Mech 68:205-224

Reichl LE (1980) A modern course in statistical physics. University of Texas Press, Austin

Sarti GC, Marrucci G (1973) Thermomechanics of dilute polymersolutions-multiple bead-spring model. Chem Eng Sci 28: 1053-1059

Sarti GC, Esposito N (1977) Testing thermodynamic constitutive equations for polymers by adiabatic deformation experiments. J Non-Newton Fluid Mech 3:65-76

Schieber JD, Venerus DC, Bush K, Balasubramanian V, Smoukov S (2004) Measurement of anisotropic energy transport in flowing polymers by using a holographic technique. Proc Natl Acad Sci U S A 101:13142-13146

Soulages J, Hütter M, Öttinger HC (2006) Thermodynamic admissibility of the extended Pom-Pom model for branched polymers. J Non-Newton Fluid Mech 139:209-213

Treloar LRG (1975) The physics of rubber elasticity (3rd ed). Clarendon, Oxford

Van Meerveld, Peters GWM, Hütter M (2004) Towards a rheological classification of flow induced crystallization experiments of polymer melts. Rheol Acta 44:119-134

Wapperom P, Hulsen MA (1998) Thermodynamics of viscoelastic fluids: the temperature equation. J Rheol 42:999-1019 